\section{Picosecond Electronics and Optoelectronics II \\ Edited by F.J. Leonberger, C.H. Lee, F. Capasso, and H. Morkoc (Springer-Verlag, 1987)}

Spectacular and simultaneous advances over the past five years in femtosecond laser technology, high-speed III-V device technology, and wideband fiber optic system components have combined to make the interaction of ultrafast optics and ultrafast electronics a cutting-edge research field in its own right. A conference entitled "Picosecond Electronics and Optoelectronics," sponsored jointly by the IEEE Lasers and Electro-Optics Society and the Optical Society of America, held its first meeting in 1985 to address these rapidly emerging research and technological possibilities. The present volume represents the proceedings of the second conference, held at the same Lake Tahoe, Nevada site, January 14-16, 1987.

The reader familiar with the first volume will recognize many of the same themes, along with reports of new emerging technologies and research areas indicative of a vital and growing field. All readers, despite some organizational difficulties stemming from the great diversity in subject areas presented, can look to this volume as a compendium of the current state and major recent developments in this field. We can identify three major themes of the volume:

1. Non-Invasive High-Speed Optical Sampling of Integrated Circuits. This is the most matured of the fields covered. Articles by Valdmanis and Pei (AT\&T) and Weingarten et al. (Stanford) review recent progress in the now standard electro-optic sampling techniques, in which picosecond or femtosecond laser pulses passively characterize electrical waveforms on ICs and transmission lines in a temporal regime ( $<1 \mathrm{ps}$ ) beyond the reach of conventional sampling oscilloscopes. Several papers report new applications of electrooptic sampling to GaAs sample-and-hold circuits, multiplexers, high-speed frequency dividers, Josephson junctions, and InP-based circuits, and evidence the expanding use of electro-optic sampling techniques in a wide variety of circuits.

Equally noteworthy, however, are several papers reporting the development of exciting new high-speed sampling techniques. May et al. (IBM) describe the coupling of a picosecond laser to a scanning electron microscope to provide a $20 \mathrm{ps}$ pulsed electron beam capable of scanning waveforms on ICs with a spatial resolution of $100 \AA$, far superior to diffraction limited light pulses. Grischkowsky et al. (IBM) demonstrate that the traditionally slower photoconductive "sliding contact" techniques can now, like their electro-optic rivals, also achieve subpicosecond resolution in both the generation and sampling of electrical waveforms. Two other IBM papers go on to discuss applications of photoconductive sampling to characterizing silicon VLSI electronics and thin film transmission line structures. Finally Weiner et al. (Bellcore) demonstrate a new photoemissive sampling technique, also capable of subpicosecond resolution, but requiring a high vacuum environment.

2. High-Speed III-V Devices and Transport Phenomena. At the device level, optics and electronics are less happily married, indeed have barely begun dating, largely because of the microscopic spatial resolution (both transverse and longitudinal) required in such experiments. The groundbreaking attempt by Mourou et al. (University of Rochester) to time-resolve velocity overshoot and resonant tunneling processes using electro-optic sampling techniques therefore constitutes a clear highlight of the volume, and will undoubtedly provide a springboard for future investigations of device performance with ultrafast optical techniques. Bar-Joseph et al. (AT\&T) describe a quite different, nontime-resolved optical characterization of the electrical state of an InGaAs MODFET using strong band-edge absorption saturation.

Other optical studies presented, however, leave devices behind and focus on material properties. Novel picosecond and femtosecond optical techniques are used to measure carrier mobility in GaAs quantum wells (Shah and Hopfel, AT\&T), bulk GaAs (Nuss and Auston, AT\&T), and across thin metal films (Brorson et al., MIT), while several other papers describe ultrafast optical spectroscopy of $\mathrm{GaAs}$ quantum wells.

Conversely, the remaining device papers leave optics behind to discuss special issues in device fabrication, performance, and physics. Cho (AT\&T) reviews progress in molecular beam epitaxy for high-speed devices, while Sollner et al. (MTT-Lincoln Lab) review recent performance results for resonant tunneling oscillators as well as new resonant tunneling devices with applications in the millimeter and submillimeter wave regime. Abe (Fujitsu) reviews progress and challenges in HEMT technology for high-performance VLSI applications. Fundamental transport physics is addressed through Monte Carlo simulations of transport in MODFETs (Hess, University of Illinois, Urbana) and hot electron relaxation in GaAs (Osman et al., Science Research Associates), while Frensley (Texas Instruments) presents a calculation of resonant tunneling response time which represents the internal state of the device by a Wigner distribution function. Finally electrical performance characteristics of an array of new high-speed devices are described.

3. High-Speed Optical Control of Devices and Circuits. Short optical pulses are also used for active generation and control of high-speed electrical signals; conversely high-speed lasers and modulators can generate and control light pulses. Taylor (Texas $A \& M)$ describes the growing practicality of using fast semiconductor lasers, fast modulators, fiber optics, and fast photoconductive detectors to generate, control, transmit, and detect signals at the multigigahertz (microwave and millimeter wave) regime, especially for military applications.

Over 20 articles then describe specific high-speed components. Bowers and Burrus (AT\&T Bell) review the state-of-theart in high-speed diode lasers and detectors, following by several articles on characterization of specific diode laser and detector systems. Gee et al. (Hughes) review design considerations for $\mathrm{LiNbO}_{3}$ and GaAs-based guided wave modulators and switches which operate at gigahertz frequencies, while Korotky et al. (AT\&T Bell) describe a Ti:LiNbO optical waveguide switch which operates up to 40 GHz. Photoconductive switches are discussed for high speed, high voltage, and/ or high repetition rate signal generation. Finally optically controlled gigahertz phase shifters, transceivers, and optoisolators are described.

Picosecond Electronics and Optoelectronics II portrays an active and growing field. The future holds probing of internal device physics with optical techniques, improved IC sampling techniques, wider applications of multigigahertz optical systems, and-almost certainly-further volumes of Picosecond Electronics and Optoelectronics. However, there are already clear signs that, in the footsteps of its famous SpringerVerlag cousin, the Picosecond Phenomena series, events will force it to be renamed "Ultrafast Electronics and Optoelectronics."

Reviewer: Michael C. Downer is assistant professor of physics, University of Texas, Austin. His research focuses on femtosecond optical techniques and their applications in condensed matter physics and microelectronic device physics. 